Pacific Journal of Mathematics

CODIMENSION ONE EMBEDDINGS OF MANIFOLDS WITH 


\section{CODIMENSION ONE EMBEDDINGS OF MANIFOLDS WITH LOCALLY FLAT TRIANGULATIONS}

\section{J. C. Cantrell}

In this note investigations are made of the problem of deciding if a given codimension one submanifold $M$ is locally flat in the ambient manifold $N$. The principal result is that if $M$ has a locally flat triangulation in which each closed simplex is locally flat in $N$, then $M$ is locally flat in $N$. This allows one to establish for simplicial homotopy manifolds certain local flatness criteria that had been previously known for $P L$ manifolds.

Our definitions are the usual ones. The reader who is unfamiliar with the subject is referred to [2] and [3] for the basic definitions and results preliminary to this note. We should mention two particular results that will be used repeatedly. The first is the following. If $D_{1}$ and $D_{2}$ are locally flat $(n-1)$-cells in $R^{n}$ that intersect in an $(n-2)$-cell that is locally flat in the boundary of each, then $D_{1} \cup D_{2}$ is locally flat [5]. The second concerns an $m$-cell $D$ in $R^{n}$, that is locally flat mod a $k$-cell $E$ that is locally flat in both $\mathrm{Bd} D$ and $R^{n}$. If $k=n-3, D$ may fail to be locally flat at points of $E$, and for $n=4, k=n-2$ it is not presently known if $D$ has to be locally flat. For all other possible values of $n, m, k$ it is known that $D$ must be locally flat [1], [3], [4], [6].

A version of the following lemma was proved in Theorem 3.2 of [2] for embeddings of cells in euclidean space. Since the result and proof are local ones, the argument given there will establish the following.

LEMma 0 . Let $M$ be an m-manifold in the interior of an $n$ manifold and let $P$ be a polyhedron in $\mathrm{Bd} M$. Assume that the following conditions are satisfied:

(a) $M-P$ is locally flat in $N$;

(b) there is a locally finite triangulation $T$ of $P$ such that each open simplex of $T$ is locally flat in $\mathrm{Bd} M$;

(c) for $\lambda$ a collaring of $\mathrm{Bd} M$ in $M$ and $\sigma$ a simplex of $T$, $\lambda($ Int $\sigma \times I)$ is locally flat in $N$. Then $M$ is locally flat in $N$.

We will say that the triangulation $T$ of the manifold $M$ is locally flat if each open simplex of $T$ is locally flat in $M$.

THEOREM 1. Let $M$ be an $(n-1)$-manifold with a locally flat triangulation $T$. Suppose that $M$ is embedded in the interior of an 
n-manifold $N$ such that each closed simplex is locally flat in $N$. Then $M$ is locally flat in $N$.

Proof. Special Case I: Int $M$ is locally flat. Since each closed simplex of $M$ is locally flat, we see that $M$ is locally flat $\bmod$ the $(n-3)$-skeleton of $\mathrm{Bd} M$.

We let $\mathrm{Bd} M$ be the polyhedron $P$ in Lemma $0, \sigma$ a $j$-simplex in $P$, and $\lambda$ a collaring of $\mathrm{Bd} M$ in $M$. Let $\left\{B_{i}\right\}_{i=1}^{\infty}$ be a monotone increasing sequence of closed $j$-cells, each locally flat in $\sigma$ (and hence in each of $M$ and $N$ ) and $\bigcup_{i=1}^{\infty} B_{i}=\operatorname{Int} \sigma$. In case $j=0$, we adopt the convention that Int $\sigma=\sigma$. We will show that $\lambda(\operatorname{Int} \sigma \times I)$ is locally flat by showing that $\lambda\left(B_{i} \times I\right)$ is locally flat for each $i$. One easily checks that $\lambda\left(B_{i} \times I\right)$ is locally flat $\bmod \lambda\left(B_{i} \times 0\right)=B_{i}$ and that $B_{i}$ is locally flat in $\mathrm{Bd} \lambda\left(B_{i} \times \mathrm{I}\right)$ and in $N$. If $j \leqq n-4$, we appeal to Theorem 1.2 of [1] to conclude that $\lambda\left(B_{i} \times I\right)$ is locally flat.

If $j=n-3$, we observe that $\lambda\left(B_{i} \times I\right)$ can be pushed into one (either) of the two $(n-2)$-simplexes in $\mathrm{Bd} M$ that has $\sigma$ as a face, and that local flatness of this simplex then implies local flatness of $\lambda\left(B_{i} \times I\right)$. This argument is written out in detail in Lemma 8 of [3].

Special Case II: $\mathrm{Bd} M$ is empty.

The proof will be downward induction on the dimension of the skeletons of $M$. Since each $(n-1)$-simplex is locally flat, it is immediate that $M$ is locally flat $\bmod$ the $(n-2)$-skeleton.

For $\sigma$ an $(n-2)$-simplex, the link of $\sigma$ in $M$ is a $o$-sphere (this follows from the fact that $M$ is a manifold) and the star of $\sigma$ is the union of two $(n-1)$-simplexes having $\sigma$ as a common face. Since each of these $(n-1)$-simplexes is locally flat, and $\sigma$ is locally flat in the boundary of each and in $M$, it follows that the star of $\sigma$ is locally flat. Thus, $M$ is locally flat at each point of Int $\sigma$.

For $\sigma$ an $(n-3)$-simplex, we again observe that, since $M$ is a manifold, the link of $\sigma$ is a 1-sphere. We let $\tau$ be a 1-simplex of $l k \sigma$ and let $B$ be the closure of $l k \sigma-\tau$. Then St $\sigma=\sigma^{*} \tau \cup \sigma^{*} B . \quad \sigma^{*} \tau$ is locally flat by hypothesis and $\sigma^{*} B$ is locally flat by Special Case I and the preceding paragraph. Hence, St $\sigma$ must be locally flat at each interior point. In particular, $M$ is locally flat at each point of Int $\sigma$.

We could continue this line of proof, viewing St $\sigma$ as the union of two locally flat cells $\sigma^{*} \tau$ and $\sigma^{*}(l k \sigma-\tau)$, as long as we knew that $l k \sigma$ was a $P L$ sphere. If $T$ were a $P L$ triangulation of $M$, we would know this for all $\sigma$, but for arbitrary triangulations we only know this for $\operatorname{dim} l k \sigma \leqq 2$ or, equivalently, $\operatorname{dim} \sigma \geqq n-4$. After this an- 
other type of proof would be needed. Fortunately these larger codimension cases have been handled (see Theorem 6.1 of [2]). For completeness, we will include the proof for the skeletons of dimension $\leqq n-4$.

Suppose then that $M$ is locally flat $\bmod$ the $j$-skeleton, $j \leqq n-4$, let $\sigma$ be a $j$-simplex and let $p$ be an interior point of $\sigma$. Let $E$ be a $j$-cell with $x \in \operatorname{Int} E \subset E \subset \sigma$ such that $E$ is locally flat in $\sigma$. Since locally flat cells lie trivially in some euclidean neighborhood [8], it is easy to find two $(n-1)$-cells $D_{1}$ and $D_{2}$ in $M$ with the following properties:

(1) $D_{1}$ and $D_{2}$ are locally flat in $M$ and intersect the $j$-skeleton only in $E$;

(2) $D_{1} \cap D_{2}=\mathrm{Bd} D_{1} \cap \mathrm{Bd} d_{2}=D_{3}$ is an $(n-2)$-cell which is locally flat in $\mathrm{Bd} D_{1}$ and $\mathrm{Bd} D_{2}$;

(3) $\left(D_{3}, E\right) \approx\left(B^{n-2}, B^{j}\right)$.

From (1) and transitivity of the relation "is locally flat in" it follows that each of $D_{1}$ and $D_{2}$ is locally flat $\bmod E$ in $N$. Then, since $\operatorname{dim} E \leqq n-4, D_{1}$ and $D_{2}$ are locally flat in $N$ and, hence, $D_{1} \cup D_{2}$ is locally flat in $N$. This implies that $M$ is locally flat at $p$. In this way we see that $M$ is locally flat $\bmod$ the $(j-1)$-skeleton, and the induction is complete.

General Case. Apply Special Case II to $M-\mathrm{Bd} M$ and then apply Special Case I to $M$.

LemMA 1. Let $\sigma$ be an (n-1)-simplex (topologically) embedded in the interior of an $n$-manifold $N, n \neq 4$, and such that Int $\sigma$ is locally flat in $N$ and each closed $(n-2)$-simplex of $\mathrm{Bd} \sigma$ is locally flat in $N$. Then $\sigma$ is locally flat.

Proof. Note that $(n-2)$-simplexes being locally flat will imply that all lower dimensional simplexes are locally flat. The proof is the same as the proof of Special Case I of Theorem 1, except that if $\operatorname{dim} B_{i}=n-2$, we get local flatness of $\lambda\left(B_{i} \times I\right)$ from Theorem 2 of [4].

CoRollary 1. Let $T$ be a locally flat triangulation of an $(n-1)$ manifold $M$ and $M$ embedded in the interior of an n-manifold $N$. If $n \neq 4$, and each open $(n-1)$-simplex and each closed $(n-2)$-simplex is locally flat in $N$, then $M$ is locally flat.

There is another interesting observation that comes from Theorem 1 and its proof. This deals with the question of local flatness of an 
( $n-1)$-sphere $S$ in $S^{n}, n \neq 4$, that is known to be locally flat $\bmod$ an $(n-2)$-cell $E$ that is locally flat in both $S$ and $S^{n}$. We can establish local flatness of $S$ as follows. We give $S$ the triangulation of the boundary of an $n$-simplex with $E$ being one of the $(n-2)$-simplexes. To meet the requirements of Corollary 1 , we need to see that each closed $(n-2)$-simplex is locally flat. Let $\sigma$ be an $(n-2)$-simplex different from $E$. Then $\sigma$ and $E$ have a common $(n-3)$-face and lie on an $(n-1)$-simplex $\tau$ that is locally flat $\bmod \mathrm{Bd} E . \quad \mathrm{As}$ in the proof of Special Case I of Theorem 1, we can push $\sigma$ across $\tau$ into the locally flat cell $E$ and conclude that $\sigma$ is locally flat.

Let $N$ be an $n$-manifold triangulated so that the link of each $i$-simplex $(0 \leqq i<n)$ has the homotopy of an $(n-i-1)$-sphere or ball. We will then call $N$ (with this triangulation) a simplicial homotopy manifold. Glaser has shown that triangulations that make $N$ a simplicial homotopy manifold are locally flat [7, Theorem 2]. This fact together with Theorem 1 and Corollary 1 gives the following extension of Theorem 12 of [3] to include simplicial homotopy manifolds.

THEOREM 2. Let $M$ be a simplicial homotopy $(n-1)$-manifold in the interior of an n-manifold $N$. (1) If $n=4$, suppose that each closed simplex of $M$ is locally flat in $N$. (2) If $n \neq 4$, suppose that each open $(n-1)$-simplex and each closed $(n-2)$-simplex of $M$ is locally flat in $N$. Then $M$ is locally flat in $N$.

In codimensions three and greater, we have a better criterion than Theorem 2 gives for codimension one embeddings. The above mentioned result of Glaser, together with Theorem 5.3 of [1] or Theorem 6.1 of [2] gives the following.

THEOREM 3. Let $M$ be an m-dimensional simplicial homotopy manifold in the interior of an n-dimensional manifold $N$ with $n-m \geqq 3$. If each open simplex of $M$ is locally flat in $N$, then $M$ is locally flat in $N$.

\section{REFERENCES}

1. J.L. Bryant and C.L. Seebeck, III, Locally nice embeddings of polyhedra, Quart

J. of Math., (Oxford) 19 (1968), 257-74.

2. J. C. Cantrell and R. C. Lacher, Local flattening of a submanifold, ibid. 20 (1969), 1-10.

3. Some local flatness criteria for low codimensional submanifolds, ibid. 21 (1970), 129-36.

4. J. C. Cantrell, T. B. Rushing and T. M. Price, $A$ class of embeddings of $S^{n-1}$ and $B^{n}$ in $R^{n}$, Proc. Amer. Math. Soc., 29 (1971), 208-10. 
5. A. V. Črnavskii, The k-stability of homeomorphisms and the union of cells, Soviet Math. Dokl., 9 (1968), 729-31.

6. P. H. Doyle, Unions of cells in En, Pacific J. Math., 10 (1960), 521-24.

7. L. C. Glaser, A proof of the most general polyhedral Schoenflies conjecture possible, Pacific J. Math., (to appear.)

8. R. C. Lacher, Locally flat strings and half strings, Proc. Amer. Math. Soc., 18 (1967), 299-304.

Received October 8, 1971 and in revised form January 6, 1972. This research was supported in part by NSF Grant GP-19961.

UNIVERSITY OF GEORGIA 



\section{PACIFIC JOURNAL OF MATHEMATICS}

EDITORS

\author{
H. SAMELSON \\ Stanford University \\ Stanford, California 94305 \\ C. R. HOBBY \\ University of Washington \\ Seattle, Washington 98105
}

\author{
J. DUGUNDJI \\ Department of Mathematics \\ University of Southern California \\ Los Angeles, California 90007 \\ RICHARD ARENS \\ University of California \\ Los Angeles, California 90024
}

\section{ASSOCIATE EDITORS}

E. F. BECKENBACH

B. H. NeUManN

F. WOLF

K. YosHIDA

\section{SUPPORTING INSTITUTIONS}

UNIVERSITY OF BRITISH COLUMBIA

CALIFORNIA INSTITUTE OF TECHNOLOGY

UNIVERSITY OF CALIFORNIA

MONTANA STATE UNIVERSITY

UNIVERSITY OF NEVADA

NEW MEXICO STATE UNIVERSITY

OREGON STATE UNIVERSITY

UNIVERSITY OF OREGON

OSAKA UNIVERSITY
UNIVERSITY OF SOUTHERN CALIFORNIA

STANFORD UNIVERSITY

UNIVERSITY OF TOKYO

UNIVERSITY OF UTAH

WASHINGTON STATE UNIVERSITY UNIVERSITY OF WASHINGTON

AMERICAN MATHEMATICAL SOCIETY NAVAL WEAPONS CENTER 


\section{Pacific Journal of Mathematics}

\section{Vol. 45, No. $1 \quad$ September, 1973}

William George Bade, Complementation problems for the Baire classes .......... 1

Ian Douglas Brown, Representation of finitely generated nilpotent groups ........ 13

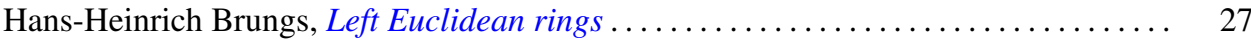

Victor P. Camillo and John Cozzens, A theorem on Noetherian hereditary rings ..... 35

James Cecil Cantrell, Codimension one embeddings of manifolds with locally flat

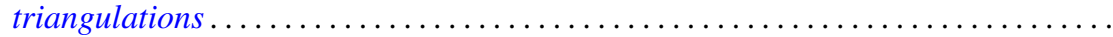

L. Carlitz, Enumeration of up-down permutations by number of rises . . . . . . . . . .

Thomas Ashland Chapman, Surgery and handle straightening in Hilbert cube

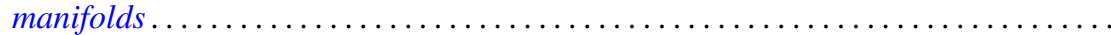

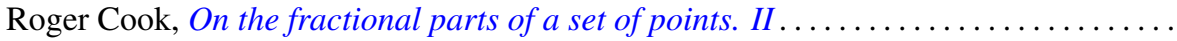

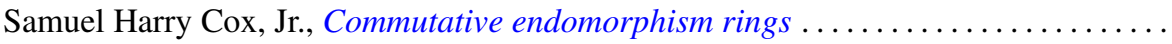

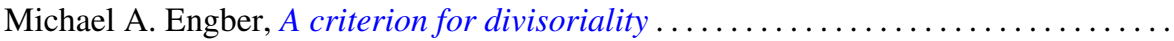

Carl Clifton Faith, When are proper cyclics injective . . . . . . . . . . . . . . 97

David Finkel, Local control and factorization of the focal subgroup . . . . . . . . . 113

Theodore William Gamelin and John Brady Garnett, Bounded approximation by

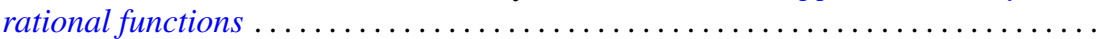

Kazimierz Goebel, On the minimal displacement of points under Lipschitzian

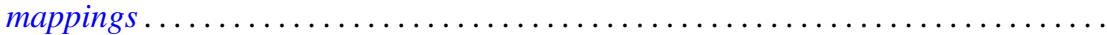

Frederick Paul Greenleaf and Martin Allen Moskowitz, Cyclic vectors for representations associated with positive definite measures: nonseparable

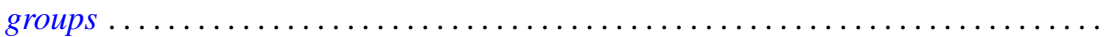

Thomas Guy Hallam and Nelson Onuchic, Asymptotic relations between perturbed linear systems of ordinary differential equations .

David Kent Harrison and Hoyt D. Warner, Infinite primes of fields and completions. .

James Michael Hornell, Divisorial complete intersections . ......

Jan W. Jaworowski, Equivariant extensions of maps ..............

John Jobe, Dendrites, dimension, and the inverse arc function .. .

Gerald William Johnson and David Lee Skoug, Feynman integrals of non-factorable

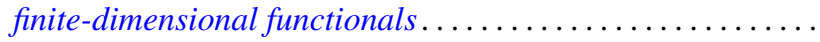

Dong S. Kim, A boundary for the algebras of bounded holomorphic functions ...... 269

Abel Klein, Renormalized products of the generalized free field and its derivatives ... 275

Joseph Michael Lambert, Simultaneous approximation and interpolation in $L_{1}$ and

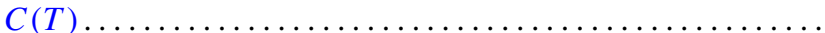

Kelly Denis McKennon, Multipliers of type $(p, p)$ and multipliers of the group

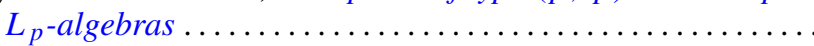

William Charles Nemitz and Thomas Paul Whaley, Varieties of implicative

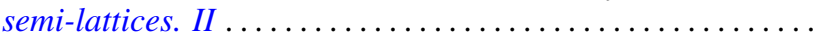

Donald Steven Passman, Some isolated subsets of infinite solvable

Norma Mary Piacun and Li Pi Su, Wallman compactifications on E-completely

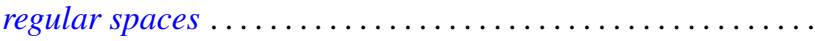

Jack Ray Porter and Charles I. Votaw, $S(\alpha)$ spaces and regular Hausdorff extensions....

Gary Sampson, Two-sided $L_{p}$ estimates of convolution transforms .

Ralph Edwin Showalter, Equations with operators forming a rig
Raymond Earl Smithson, Fixed points in partially ordered sets .

Victor Snaith and John James Ucci, Three remarks on symmetric products and

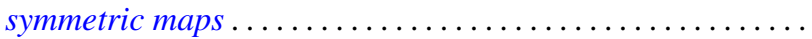

